\title{
Detección de residuos de quinolonas en carne bovina de venta en mercados municipales de la ciudad de Guatemala
}

\author{
Quinolone antibiotic residues in beef meat sold in municipal markets of Guatemala City

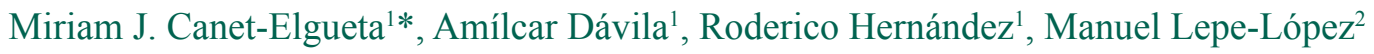 \\ ${ }^{1}$ Departamento de Investigación y Extensión, Facultad de Medicina Veterinaria y Zootecnia, \\ Universidad de San Carlos de Guatemala; ${ }^{2}$ PhD Program in Conservation Medicine, Facultad de Ciencias de la Vida, \\ Universidad Andrés Bello, Santiago, Chile.
}

*Autor al que se dirige la correspondencia: miriamjcanet@hotmail.com

Recibido: 30 de noviembre 2017 / Revisión: 07 de marzo 2018 / Aceptado: 09 de octubre 2018

\section{Resumen}

T a resistencia bacteriana es una amenaza creciente para la salud pública mundial. El mal uso y uso excesivo de Lantimicrobianos en personas y animales está acelerando este proceso. En el caso de residuos antimicrobianos en productos de origen animal, se sabe que producen reacciones alérgicas, así como inducción de resistencia en bacterias transmitidas por alimentos como Salmonella, Campylobacter y Escherichia coli. Este estudio tuvo como objetivo detectar la presencia de residuos de quinolonas en carne bovina de venta en mercados municipales de la ciudad de Guatemala. Se obtuvieron 161 muestras para análisis. La detección de quinolonas se realizó por metodología de Inmunoensayo Asociado a Enzima (Elisa). Siete de 161 (5\%) contenían residuos de quinolonas. Los niveles medios (desviación estándar) detectados fueron 16.497(1.69) $\mu \mathrm{g} / \mathrm{kg}$; ningún límite fue superior al límite máximo residual permitido $(100 \mu \mathrm{g} / \mathrm{kg})$. Este estudio indicó que existen residuos de antibiótico en algunas carnes de res en Guatemala.

Palabras claves: Circulación, antibióticos, alimentos, resistencia

\begin{abstract}
$\mathrm{A}$ ntimicrobial resistance is an increasingly serious threat to global public health. The misuse and overuse of antimicrobials in people and animals is an accelerating factor for this process. In the case of antimicrobial residues in animal origin products, it is known that they cause allergic reactions as well as induction of resistance in bacteria such as Salmonella, Campylobacter and Escherichia coli. This study aimed to find the presence of quinolones residues in beef meat. A total of 161 samples were collected from municipal markets in Guatemala City for analysis. Extraction and determination of quinolones was performed by Enzyme-linked Immunoassay (Elisa) methodology. Seven of 161 (5\%) contained quinolone residues. The mean levels $( \pm$ SE) of quinolones were $16.497(1.69) \mu \mathrm{g} / \mathrm{kg}$; no limit was superior than the maximum residual limit allowed (100 $\mu \mathrm{g} / \mathrm{kg})$ by FDA. Even though none of the samples exceeded the upper limit it is important that some residues of quinolone were found.
\end{abstract}

Keywords: Circulation, antibiotics, resistance, food 


\section{Introducción}

La resistencia bacteriana es una emergencia pública mundial y representa una amenaza a la salud humana y animal, generando una mayor morbilidad, mortalidad, estancia hospitalaria prolongada, así como fuertes repercusiones económicas para el sistema sanitario (Casellas, 2011). El principal factor de riesgo para el desarrollo de resistencia bacteriana es el uso inadecuado y abuso de los antibióticos en medicina humana y veterinaria (Meek, Vyas, \& Piddock, 2015). En el sector de la producción animal los antimicrobianos son utilizados como promotores de crecimiento, con fines terapéuticos y profilácticos (Grande, Falcón, \& Gándara, 2000) siendo los principales grupos las tetraciclinas, beta lactámicos, macrólidos, quinolonas y lincosaminas. Los antibióticos utilizados como promotores de crecimiento suponen un mayor riesgo ya que los mismos son utilizados por períodos prolongados de tiempo en concentraciones sub terapéuticas facilitando la presión selectiva de bacterias resistentes (Landers, Cohen, Wittum, \& Larson, 2012). Los residuos de estas sustancias o sus metabolitos en la carne y otros alimentos de origen animal pueden causar efectos adversos en la salud de los consumidores (Landers et al., 2012).

Las quinolonas merecen un mayor escrutinio ya que no solamente pueden tener un efecto directamente tóxico y producir reacciones de hipersensibilidad, sino que además están asociadas a una mayor inducción de resistencia en bacterias transmitidas por alimentos como Salmonella, Campylobacter y Escherichia coli (Subbiah, Mitchell, \& Call, 2016). Las mutaciones bacterianas ante el uso de quinolonas pueden ocurrir rápidamente, incluso durante la terapia antimicrobiana. El uso excesivo y continuo de tan solo un antibiótico perteneciente a esta familia de antimicrobianos puede generar resistencia a todo el grupo de quinolonas, limitando su efectividad en un futuro cercano (Bennett, Dolin, \& Blaser, 2014).

La detección de residuos de quinolonas en diferentes productos de origen animal, desde carnes de animales terrestres y acuícolas hasta productos de miel de abeja (Chang, Wang, \& Tsai, 2010; Karbiwnyk, Carr, Turnipseed, Andersen, \& Miller, 2007; van Vyncht et al., 2002; Zhao, Li, Jiang, Li, \& Shen, 2007), ha incrementado la preocupación en países de la Unión Europea, China y Estados Unidos resultando incluso en la prohibición del uso de enrofloxacina en varios sistemas de producción, tal como el caso de la avicultura (Food and Drug Administration, 2005).
La seguridad alimentaria exige la vigilancia y control de sustancias y residuos, tanto de origen animal como vegetal, en toda la cadena alimentaria desde la producción primaria hasta la distribución final (Garzón, 2009). Dentro de las técnicas existentes para la detección de residuos de antibiótico se destacan el empleo de biosensores por inmuno absorción ligado a enzimas (Elisa), así como cromatografía de gases (GC), cromatografía líquida de alta resolución (HPLC) y cromatografía de gases acoplada a la espectrometría de masas (GC/MS) (Talero-Pérez, Medina, \& Rozo-Núñez, 2014). El límite máximo residual (LMR) se define como aquella concentración aceptable de una sustancia en los tejidos comestibles de un animal (músculos, hígado, riñones, grasa, leche, miel y huevos) y que al ser ingerida por el ser humano no constituye ningún riesgo para su salud. Los LMR se fijan para cada especie animal y para cada tejido siendo el LMR permitido de quinolonas en el músculo de carne de vacuno de 100 $\mu \mathrm{g} / \mathrm{kg}$ de acuerdo a lo establecido por la Administración de Alimentos y Medicamentos (FDA) de Estados Unidos y la Autoridad Europea de Seguridad Alimentaria (EFSA) (Garzón, 2009).

En Guatemala no existen leyes que regularicen el uso de antimicrobianos en medicina humana y veterinaria. No obstante, la Organización Mundial de la Salud (OMS) y la Organización de Salud Animal (OIE) han ofrecido directrices sobre el buen uso de antimicrobianos bajo el enfoque de 'una salud', dada la interrelación entre salud humana y salud animal. Estas estrategias internacionales priorizan entre sus objetivos mejorar la vigilancia y la investigación de la disponibilidad de residuos en alimentos.

El presente estudio exploró la circulación de quinolonas en carne de bovino expendida en mercados municipales de la ciudad capital respondiendo a dos preguntas de investigación: ¿Existe circulación de quinolonas en carne de bovino comercializada en los mercados municipales de ciudad de Guatemala? y de existir tales residuos, ¿Las cantidades de quinolonas circulantes son cercanas al límite máximo residual? Se espera que las respuestas a estas preguntas colaboren a entender la disponibilidad de este importante grupo de antimicrobianos en carne bovina expendida en Guatemala, dada la amenaza que la resistencia bacteriana representa para la salud humana.

\section{Presentación del caso}

Se realizó un muestreo no probabilístico por conveniencia recolectándose un total de 161 muestras de 
carne corte costillar de los 23 mercados municipales ubicados en la ciudad de Guatemala durante los meses de junio y julio del 2017. Se analizó costillar por ser útil para monitoreo a bajo costo. Fueron muestreados la totalidad de puestos de carne en cada mercado municipal. Se preguntó a los vendedores de carne si conocían el origen del rastro donde procedía la carne. Las muestras fueron identificadas con lugar y fecha y conservadas en una hielera para preservar una temperatura de $7^{\circ} \mathrm{C}$. Inmediatamente después de la recolección, las muestras fueron trasladadas al Laboratorio de Desarrollo de Soluciones Globales (DSG) en donde se realizó el análisis.

Los residuos de quinolonas se determinaron mediante la metodología Elisa usando la prueba $\mathrm{Ri}$ dascreen Quinolones/Elisa ${ }^{\circledR}$ (R-Biopharm AG, Darm stadt, Alemania). El ensayo se realizó de acuerdo con las directrices del fabricante con un único análisis por cada una de las muestras. La prueba mostró especificidad a ciprofloxacina (100\%), norfloxacina, enrofloxacina, marbofloxacina, danofloxacina, difloxacina, flumequin, ofloxacina (> 100\%), sarafloxacina (43\%) y ácido oxolínico (24\%). El límite inferior de detección de la prueba es de $10 \mathrm{ppb}(10 \mu \mathrm{g} / \mathrm{kg})$.

Los datos fueron tabulados en MS Excel analizándose mediante el software XL STAT. Para las variables categóricas se realizó descripción de frecuencias y porcentajes y para las variables continuas, se estimó media y desviación estándar.

Siete muestras resultaron positivas a residuos de quinolonas, equivalente al $5 \%$ del total de carne de bovino analizada $(\mathrm{n}=161)$. Los niveles medios (desviación estándar) de residuos de quinolonas fueron de $16.49(1.69) \mu \mathrm{g} / \mathrm{kg}$ en muestras positivas. Ninguna de las muestras presentó un límite superior al límite máximo residual permitido $(100 \mu \mathrm{g} / \mathrm{kg})$. Otras 80 muestras resultaron poseer trazas de quinolonas.

En relación a la ubicación de los rastros de donde provienen las muestras de carne, los vendedores informaron que 58/161 (36 \%) ignora su origen, 38/161 (24\%) proviene de Petén y 14/161 (9\%) de Izabal y Chinautla, respectivamente. El resto de muestras provenían de Palencia, Retalhuleu, Jocotales, Villa Nueva, San José Pinula y Escuintla. En $11 \%$ de los casos los vendedores se negaron a proporcionar el origen de la carne. Del total de muestras que provienen de Petén, $5 / 38(13 \%)$ fueron positivas para residuos de quinolonas. Del total de muestras positivas, 5/7 (71\%) provenían de rastros del departamento de Petén.

\section{Discusión}

Los residuos de antibióticos en los alimentos de origen animal suponen un problema de salud pública y un riesgo para la salud humana, ya que éstos han sido relacionados con reacciones de hipersensibilidad, así como inducción de cepas resistentes (Nisha, 2008). Teniendo en cuenta la importancia y las estrictas regulaciones en seguridad alimentaria, en muchos países se realizan periódicamente muestreos de residuos de antibióticos en alimentos como aves de corral, ganado y leche (Garzón, 2009). Respecto a residuos de antibiótico en ganado, en Turquía, Kaya, Yavuz, Akar, Liman y Filazi (1992) informaron que la tasa de residuos de antibióticos en bovino era del $18 \%$ (15/85 muestras analizadas). En Sudán, 52/300 (17\%) bovinos mostraron resultados positivos para la presencia de antibióticos en uno o más órganos en muestras post mortem (riñón 10\%, hígado $7.66 \%$ y músculo $3 \%$ ) (Alla, Mohamed, \& Abdelgadir, 2013). En Ghana, la prevalencia total de residuos de antibióticos en alimentos de origen animal fue del $21 \%$ en 634 muestras analizadas (30 $\%$ en carne de res) (Donkor et al., 2011). Otro estudio demostró que 60/104 (57\%) muestras de carne de res fueron positivas para quinolonas con niveles medios (desviación estándar) de $6.64(1.11) \mu \mathrm{g} / \mathrm{kg}$ (Er et al., 2013). En Egipto de 600 bovinos analizados, $2 \%$ presentó resultado positivo para residuos de oxitetraciclinas; $1 \%$ por encima del LMR permitido (Morshdy, El-Atabany, Hussein, \& Darwish, 2013). Omotoso y Omojola (2015) demostraron que mediante análisis microbiológico el 50, 55 y $40 \%$ de las carnes de res, pollo y cerdo, respectivamente, fueron positivos para la presencia de residuos de antibióticos. En Vietnam se llevó a cabo un plan de seguimiento de antibióticos residuales en alimentos de origen animal entre 2012 y 2013 obteniendo un $7 \%$ de positividad en las muestras de res analizada (Yamaguchi et al., 2015).

En Guatemala, no se conocían hasta ahora datos de la circulación de residuos de quinolonas en bovinos. En comparación con lo reportado en la literatura, se evidencia un menor porcentaje de positividad de detección de residuos de quinolonas ( $5 \%$ ). Esto puede deberse a múltiples factores entre los que cabe mencionar el alto costo de las quinolonas en el mercado y su mayor utilización en el sector avícola comparado con el sector ganadero. Por otra parte, estos resultados están sujetos a la cantidad de muestras obtenidas y a la carencia de repeticiones temporales y espaciales. Es preocupante 
que se encontraron residuos de quinolonas únicamente con una muestra por sitio, sugiriendo que la probabilidad de encontrar residuos con muestreos más extensos sería mucho mayor a lo presentado en estos datos. Estudios realizados en el país han demostrado la presencia de cepas de Salmonella sp y Campylobacter sp aisladas en carne de pollo resistentes a enrofloxacina en un 52 y $59 \%$, respectivamente, cifra mayor a la reportada en el continente americano y otros continentes (Jarquín et al., 2015) demostrando de manera indirecta la utilización de quinolonas en el ámbito avícola veterinario.

Si bien la presencia de residuos de quinolonas fue baja en este estudio, debe considerarse que sean otros grupos de antimicrobianos los que se encuentren circulando en mayor cantidad, por lo que estudios que consideren su tamizaje son necesarios (Draisci et al., 2001). En Guatemala la presencia de residuos de antibióticos en productos para consumo humano ha sido estudiada; Martínez (2009) demostró que $12 \%$ de 48 muestras de leche procedentes de 16 fincas ganaderas ubicadas en ocho departamentos (Chimaltenango, Chiquimula, Escuintla, Guatemala, Jutiapa, Santa Rosa, Suchitepéquez y Zacapa) contenían residuos de beta-lactámicos y tetraciclinas en tanto que Ramírez y Miranda (2015) encontraron tetraciclina en pechugas de pollo procedentes de una granja avícola ubicada en ciudad de Guatemala con niveles por encima del LMR permitido.

Nuestros resultados están basados en las capacidades del método Elisa, existiendo otras técnicas con mayor sensibilidad y especificidad para la búsqueda de residuos de antimicrobianos, de alto costo y poca disponibilidad en Guatemala, como la cromatografía líquida y la espectrofotometría de masa (Blasco, Picó, \& Torres, 2007). No obstante el método Elisa es recomendado como una forma de tamizaje de muestras de una población carente de exploración, como en el presente caso que los esfuerzos no fueron dirigidos a un ingrediente activo particular (enrofloxacina) sino más bien a la presencia de una familia de antibióti$\cos$, dado que las reacciones cruzadas entre diferentes antibióticos relacionados estructuralmente otorgaron un aumento de la probabilidad de obtener un resultado positivo de las muestras (Aga et al., 2005). Lo anterior hace necesario continuar los esfuerzos de investigación de este grupo de antibióticos de forma singular, según los antimicrobianos con alta demanda comercial para el área de veterinaria en Guatemala.

Las bajas cantidades de residuos encontrados podrían ser explicados además por un cumplimiento en el tiempo de retiro de estos fármacos, ya sea por parte del productor o por exigencia del personal de rastro. Es importante tomar en cuenta que la ubicación de los rastros de donde provenían las carnes fue proporcionada por el vendedor al momento de la toma de muestra, limitando así la confiabilidad del dato. Cabe resaltar que una tercera parte de las personas que ofrecen carne de bovino en los mercados municipales de ciudad de Guatemala ignoran la procedencia del producto. Lo anterior es preocupante en términos de inocuidad de los alimentos, debido a que la trazabilidad es una práctica que debería ser habitual ofreciendo acceso a la información de la ubicación del origen y la trayectoria del producto hasta el consumidor final (Neira, 2003). Sin embargo, los esfuerzos gubernamentales de trazabilidad bovina se implementaron en Guatemala a partir del 2014 siendo éstos a la fecha inefectivos y por lo tanto poco útiles para medir resultados (Leal, 2016).

Por otra parte, el desconocimiento de la procedencia de la carne analizada podría ser explicado por la posibilidad de contrabando de carne de bovino al país, similar a otras actividades de este tipo consolidadas en Guatemala (Galemba, 2012). Es sabido que la mayoría de fincas productoras de ganado bovino del país se encuentran establecidas en la zona norte (Pérez, Holmann, \& Schuetz, 2006), por lo que esto explicaría la cantidad de muestras positivas procedentes de Petén en nuestro análisis.

En el país existen varios rastros que no son aprobados por el Ministerio de Agricultura Ganadería y Alimentación, debilitando el control, calidad e inocuidad del producto. Estudios que sean dirigidos a Petén deben realizarse considerando que es el departamento con mayor producción de ganado en el país. Lo anterior significaría que en dicha área se estén utilizando antimicrobianos de menor espectro y bajo costo diferente a las quinolonas, con el fin de reducir el impacto económico ante los desafíos que esta actividad presenta para Centroamérica (Guillen, Pomareda, Umaña, \& Pérez, 1998).

Se considera que las muestras de carne obtenidas durante el período de estudio excluyen los riesgos para la salud en términos de la presencia de quinolonas, aunque se desconocen datos de la exposición crónica a estos residuos. En términos de prevención de la resistencia a los antibióticos en humanos, la baja cantidad de residuos de quinolonas observada en este estudio representa un resultado positivo. Debe sin embargo mantenerse un estricto control y monitoreo en el uso 
de antimicrobianos en el sector veterinario debido a que en el país no existen políticas de Estado ni leyes que regulen su uso controlado. Los resultados obtenidos son válidos para una visión transversal del problema; empero, los resultados pueden ser de mayor valor ante un enfoque longitudinal, y, por lo tanto, ya verificada la circulación de quinolonas son necesarios los esfuerzos en monitoreo continuo para prevenir niveles cercanos al LMR.

La verificación en la circulación de quinolonas en productos de origen animal en ciudad de Guatemala abre la discusión sobre el uso de antimicrobianos en la ganadería guatemalteca, debido a la escasez de estudios en este tema y a la necesidad de incrementar los esfuerzos de investigación sobre los antimicrobianos y otros fármacos utilizados en la industria cárnica (e.g. clembuterol).

\section{Agradecimientos}

Esta investigación fue cofinanciada por Digi-Usac-2017, Proyecto: 4.8.63.1.02. Se agradece también a Wilson Valdez y a Luis Morales por sus comentarios y sugerencias para redactar la propuesta ingresada a la DIGI para obtener el financiamiento.

\section{Referencias}

Aga, D. S., O’Connor, S., Ensley, S., Payero, J. O., Snow, D., \& Tarkalson, D. (2005). Determination of the persistence of tetracycline antibiotics and their degradates in manure-amended soil using enzyme-linked immunosorbent assay and liquid chromatography- mass spectrometry. Journal of Agricultural and Food Chemistry, 53(18), 71657171. doi:10.1021/jf050415+

Alla, M. B. W., Mohamed, T. E., \& Abdelgadir, A. E. (2013). Detection of antibiotics residues in beef in Ghanawa Slaughterhouse, Khartoum State, Sudan. African Journal of Food Science, 5(10), 574-580.

Bennett, J. E., Dolin, R., \& Blaser, M. J. (2014). Mandell, Douglas, and Bennett's Principles and Practice of Infectious Diseases ( $8^{\mathrm{a}}$ edición). Elsevier Health Sciences.
Blasco, C., Picó, Y., \& Torres, C. M. (2007). Progress in analysis of residual antibacterial in food. Trends in Analytical Chemistry, 26(9), 895-913. doi: 10.1016/j.trac.2007.08.001

Casellas, J. M. (2011). Antibacterial drug resistance in Latin America: Consequences for infectious disease control. Revista Panamericana de Salud Pública, 30(6), 519-528.

Chang, C. S., Wang, W. H., \& Tsai, C. E. (2010). Simultaneous determination of 18 quinolone residues in marine and livestock products by liquid chromatography/tandem mass spectrometry. Journal of Food and Drug Analysis, 18(2), 87-97.

Donkor, E. S., Newman, M. J., Tay, S. C., Dayie, N. T., Bannerman, E., \& Olu-Taiwo, M. (2011). Investigation into the risk of exposure to antibiotic residues contaminating meat and egg in Ghana. Food Control, 22(6), 869-873. doi:10.1016/j.foodcont.2010.11.014

Draisci, R., i Quadri, F. d., Achene, L., Volpe, G., Palleschi, L., \& Palleschi, G. (2001). A new electrochemical enzyme-linked immunosorbent assay for the screening of macrolide antibiotic residues in bovine meat. Analyst, 126(11), 19421946.

Er, B., Onurdağ, F. K., Demirhan, B., Özgacar, S. Ö., Öktem, A. B., \& Abbasoğlu, U. (2013). Screening of quinolone antibiotic residues in chicken meat and beef sold in the markets of Ankara, Turkey. Poultry Science, 92(8), 22122215. doi:10.3382/ps.2013-03072

Food and Drug Administration (July 28, 2005). FDA Announces Final Decision About Veterinary Medicine. Recuperado de http://m.bfr-mealstudie.de/cm/343/fda_announces_final_decision_ about_veterinary_medicine.pdf

Galemba, R. (2012). Taking contraband seriously: Practicing "legitimate work" at the MexicoGuatemala border. Anthropology of Work Review, 33(1), 3-14. doi:10.1111/j.1548-1417.2012. 01072.x

Garzón, T. (2009). La inocuidad de alimentos y el comercio internacional. Revista Colombiana de Ciencias Pecuarias, 22(3), 330-338.

Grande, B. C., Falcón, M. G., \& Gándara, J. S. (2000). El uso de los antibióticos en la alimentación 
animal: perspectiva actual. The use of antibiotics in animal feeds: An actual perspective. CyTAJournal of Food, 3(1), 39-47.

Guillen, R., Pomareda, C., Umaña, V., \& Pérez, E. (1998). Desafíos y oportunidades para la ganadería e industrias afines, en Centroamérica. Costa Rica: Unidad Regional de Asistencia Técnica.

Jarquín, C., Álvarez, D., Morales, O., Morales, A. J., López, B., Donado, P., ... Doyle, M. P. (2015). Salmonella on raw poultry in retail markets in Guatemala: Levels, antibiotic susceptibility, and serovar distribution. Journal of Food Protection, 78(9), 1642-1650. doi: 10.4315/0362-028X.JFP15-117

Karbiwnyk, C. M., Carr, L. E., Turnipseed, S. B., Andersen, W. C., \& Miller, K. E. (2007). Determination of quinolone residues in shrimp using liquid chromatography with fluorescence detection and residue confirmation by mass spectrometry. Analytica Chimica Acta, 596(2), 257-263. doi:10.1016/j.aca.2007.06.018

Kaya, S., Yavuz, H., Akar, F., Liman, B. C. \& Filazi, A. (1992). Mezbahadan sağlanan sığır et, karaciğer ve bobrek orneklerinde antibiyotik kalıntıları. Ankara Üniversity Veteriner Fakültesi Dergisi, 39(1-2), 13-29.

Landers, T. F., Cohen, B., Wittum, T. E., \& Larson, E. L. (2012). A review of antibiotic use in food animals: Perspective, policy, and potential. Public Health Reports, 127(1), 4-22

Leal, L. A. (2016). Trazabilidad de productos de ganado bovino. Revista Análisis de la Realidad Nacional, 5(102), 64-74

Martínez, D. E. (2009). Determinación de residuos de antibióticos beta lactámicos y tetraciclinas en leche cruda en productores de COPROLECHE (Tesis de licenciatura). Universidad de San Carlos de Guatemala, Facultad de Veterinaria, Guatemala.

Meek, R. W., Vyas, H., \& Piddock, L. J. V. (2015). Nonmedical uses of antibiotics: Time to restrict their use? PLoS Biology, 13(10). e1002266. doi: 10.1371/journal.pbio.1002266

Morshdy, A. E., El-Atabany, A. I., Hussein, M. A., \& Darwish, W. S. (2013). Oxytetracycline residues in bovine carcasses slaughtered at Mansoura Abattoir, Egypt. Japanese Journal of Veterinary Research, 61, S44-S47.

Neira, M. (2003). En qué dirección va la seguridad alimentaria. Revista Española de Salud Pública, 77(3), 307-311.

Nisha, A. R. (2008). Antibiotic residues-A global health hazard. Veterinary World, 1(12), 375-377.

Omotoso, A. B., \& Omojola, A. B. (2015). Fluoroquinolone residues in raw meat from open markets in Ibadan, Southwest, Nigeria. International Journal of Health, Animal Science and Food Safety, 2(1), 32-40. doi:10.13130/2283-3927/4739

Pérez, E., Holmann, F. J., \& Schuetz, P. (2006). Evolución de la ganadería bovina en países de América Central: Costa Rica, Guatemala, Honduras y Nicaragua (Documento de trabajo no. 205). Cali, Colombia: Centro Internacional de Agricultura Tropical e International Livestock Research Institute.

Ramírez, B. A. O., \& Miranda, R. V. (2015). Determinación de residuos de tetraciclina en carnes de pollo que se consumen en la ciudad de Guatemala. Revista Científica de la Facultad de Ciencias Químicas y Farmacia, 11(1), pp. 1-5.

Subbiah, M., Mitchell, S. M., \& Call, D. R. (2016). Not all antibiotic use practices in food-animal agriculture afford the same risk. Journal of Environmental Quality, 45(2), 618629. doi:10.2134/jeq2015.06.0297.

Talero-Pérez, Y. V., Medina, O. J., \& Rozo-Núñez, W. (2014). Técnicas analíticas contemporáneas para la identificación de residuos de sulfonamidas, quinolonas y cloranfenicol. Universitas Scientiarum, 19(1), 11-28.doi:10.11144/ Javeriana.SC19-1.taci

van Vyncht, G., Jànosi, A., Bordin, G., Toussaint, B., Maghuin-Rogister, G., De Pauw, E., \& Rodriguez, A. R. (2002). Multiresidue determination of (fluoro) quinolone antibiotics in swine kidney using liquid chromatography-tandem mass spectrometry. Journal of Chromatography A, 952(1-2), 121-129. doi: 1016/S00219673(02)00092-4 
Yamaguchi, T., Okihashi, M., Harada, K., Konishi, Y., Uchida, K., Do, M. H. N., ... Dao, K. T. V. (2015). Antibiotic residue monitoring results for pork, chicken, and beef samples in Vietnam in 2012-2013. Journal of Agricultural and Food Chemistry, 63(21), 5141-5145. doi:10.1021/ jf505254y
Zhao, S. J., Li, C., Jiang, H. Y., Li, B. Y., \& Shen, J. Z. (2007). Simultaneous determination of 7 quinolones residues in animal muscle tissues by high performance liquid chromatography. Chinese Journal of Analytical Chemistry, 35(6), 786-790. doi:10.1016/S1872-2040(07)60056-5 